1 Dissociable neural effects of temporal expectations due to passage of time and contextual

2

3

4

5

6

8 Netherlands

$9 \quad{ }^{3}$ Max Planck Institute for Empirical Aesthetics, Frankfurt am Main, Germany

${ }^{4}$ Department of Biomedical Sciences, City University of Hong Kong, Hong Kong

11

Corresponding authors: rauksztu@ @ityu.edu.hk; todorovic.ana@gmail.com

12

\section{PRESENT ADDRESS}

Ryszard Auksztulewicz, Department of Biomedical Sciences, City University of Hong Kong, 31 Tat Chee Avenue, Kowloon Tong, Hong Kong S.A.R.

Ana Todorovic, Oxford Centre for Human Brain Activity (OHBA), University Department of Psychiatry, Warneford Hospital, Oxford OX3 7JX, United Kingdom

\section{HIGHLIGHTS}

- Predictability of tone onset times affects auditory network connectivity

- Foreperiod and distribution of events in time have dissociable neural substrates

- Decreased prediction error at different levels of cortical hierarchy

\section{ACKNOWLEDGMENTS}

We would like to thank Kia Nobre and Floris de Lange for their helpful comments on experimental design and data analysis. R.A. is funded by the European Commission's Marie Skłodowska-Curie Global Fellowship (750459). 


\section{ABSTRACT}

The human brain is equipped with complex mechanisms to track the changing probability of events in time. While the passage of time itself usually leads to a mounting expectation, context can provide additional information about when events are likely to happen. In this study we dissociate these two sources of temporal expectation in terms of their neural correlates and underlying brain connectivity patterns. We analysed magnetoencephalographic (MEG) data acquired from $\mathrm{N}=24$ healthy participants listening to auditory stimuli. These stimuli could be presented at different temporal intervals but occurred most often at intermediate intervals, forming a contextual probability distribution. Evoked MEG response amplitude was sensitive to both passage of time and contextual probability, albeit at different latencies: the effects of passage of time were observed earlier than the effects of context. The underlying sources of MEG activity were also different across the two types of temporal prediction: the effects of passage of time were localised to early auditory regions and superior temporal gyri, while context was additionally linked to activity in inferior parietal cortices. Finally, these differences were modelled using biophysical (dynamic causal) modelling: passage of time was explained in terms of widespread gain modulation and decreased prediction error signalling at lower levels of the hierarchy, while contextual expectation led to more localised gain modulation and decreased prediction error signalling at higher levels of the hierarchy. These results present a comprehensive account of how independent sources of temporal prediction may be differentially expressed in cortical circuits.

\section{KEYWORDS}

Temporal prediction; Predictive coding; Hazard rate; Auditory processing; Magnetoencephalography; Dynamic causal modelling 
INTRODUCTION

61

If we know that we will hear a tone between this instant and a fixed future moment, but not exactly when, then, as we wait for the tone to arrive, its probability of occurring at the next instant will grow. The possible onset times of the tone might also have a particular temporal distribution, for example, tones might be more frequent within a narrow time window. The probability of such a tone occurring at a given moment will be a combination of the distribution of onset times and the probability of tone onset increasing over time (Nobre, Correa \& Coull, 2007; Janssen \& Shadlen, 2005). This combined computation is known as the hazard rate.

The brain tracks temporal hazard rates using a wide network of areas. Mounting temporal expectation correlates with BOLD activity in early sensory cortex, the cerebellum, as well as fronto-parietal regions including the inferior parietal cortex (IPC), supplementary motor area (SMA), and frontal areas (Bueti, et al., 2010). Electrophysiological studies have also identified that early sensory regions (Ghose \& Maunsell, 2002), the SMA (Akkal, et al., 2004), and lateral intraparietal cortex (Janssen \& Shadlen, 2005; Leon \& Shadlen, 2003) are all sensitive to temporal expectation.

While temporal expectation modulates neuronal activity over multiple regions including sensory and frontal cortices, other areas, such as the superior temporal gyrus (STG), SMA, and IPC may be more sensitive to the final integration of passage of time and contextual information (Muller \& Nobre, 2014). For example, one study found that activity only in prefrontal, but not in sensory regions, could be linked with faster reaction times in the presence of temporal expectation (Vallesi, et al., 2009). A different study found that while the superior temporal gyri (STG), SMA, and prefrontal regions were all involved in time estimation, only the STG was involved in estimating the duration of one stimulus in comparison to another stimulus (Coull, et al., 2008), showing additional sensitivity to context. A similar dissociation has been found more recently within the frontoparietal network, where the IPC showed sensitivity to fixed contextual temporal probabilities while activity in the intraparietal sulcus and inferior frontal regions was modulated by hazard rate (Coull, et al., 2016).

Apart from estimating which brain areas are involved in tracking hazard rates, an important question is how they communicate in the process. A large body of literature finds that when stimulus events are predictable, less neural activity is needed to process them (Kok, Jehee, and 
de Lange 2012; Wacongne, et al. 2011; Todorovic, et al. 2011), and this includes predictability of stimulus occurrence in time (Alink, et al. 2010; Costa-Faidella, et al. 2011; Fischer, Plessow, and Ruge 2013; Lange 2013). Predictive coding models posit that this decrease in neural activity rests on recurrent message passing between areas along the cortical hierarchy (Friston 2005; Lee and Mumford 2003; Spratling 2010), where less predictable stimuli would correspond to a network with more feedforward activation (corresponding to prediction errors being sent forward), less feedback inhibition (corresponding to predictions being sent back) and less intrinsic inhibition (corresponding to the gain or precision of prediction errors).

In this study we asked whether the passage of time and the distribution of events in time are jointly tracked in the brain as a unitary probabilistic computation, or whether, alternatively, they correspond to two probabilistic computations with different correlates in the auditory network. We additionally looked at how the brain areas involved in these two elements of tracking the hazard rate mutually interact. We found that temporal expectation of tones could be localised to sources corresponding to the primary auditory cortex (A1), superior temporal gyrus (STG) and inferior parietal cortex (IPC). The hypothesis of two separate probabilistic computations better described our data, with passage of time having an earlier effect than temporal distribution of events, as well as affecting connectivity in a broader network, that partially diverged from predictive coding models.

\section{MATERIALS AND METHODS}

\subsection{Participants}

Twenty-five healthy participants (17 female, mean \pm SD age $23.7 \pm 7.8$ years) enrolled in the study upon written informed consent, and one was later excluded due to excessive measurement noise. All experimental procedures were approved by the local ethics committee (Committee on Research Involving Human Subjects, Region Arnhem-Nijmegen, The Netherlands) and were conducted in accordance with the Declaration of Helsinki. Participants had normal hearing, normal or corrected-to-normal vision, and no history of neurological or psychiatric disorders. Data from one participant were excluded from analysis due to excessive artefacts in MEG recordings (>30\% trials). 

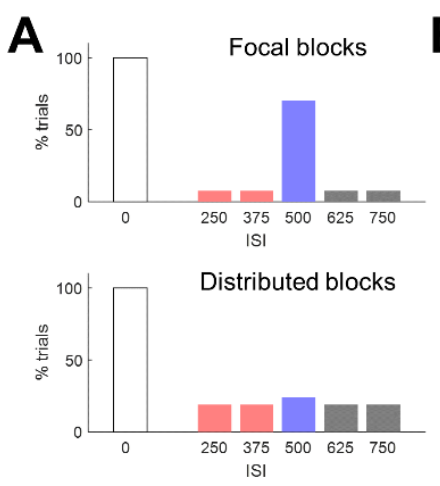

B

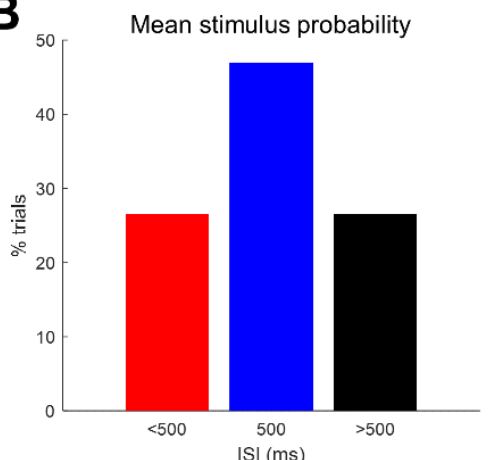

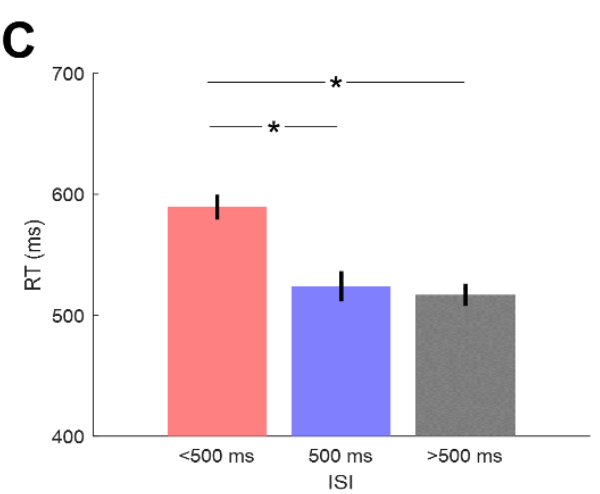
a deviant tone.

In addition to the influence of passage of time on temporal expectations (i.e., the longer the

Figure 1. Experimental stimuli and behavioural results. (A) Following a first tone marking the beginning of a trial (white bar), another tone was presented at a variable inter-stimulus interval (ISI). In focal (distributed) blocks, the second tone was delivered at ISI $=500 \mathrm{~ms}$ with $70 \%$ (24\%) probability among five possible ISIs. Occasionally, either of the two tones was presented with a deviant pitch; participants were instructed to detect these deviants with a speeded button press. (B) Averaging across focal and distributed blocks, the probability of delivering the second tone at ISI $=500 \mathrm{~ms}$ was higher (47\%) relative to shorter or longer ISIs (26.5\%). (C) Effects of ISI on RTs: deviant tones presented at short latencies $(<500 \mathrm{~ms})$ were detected slower than deviant tones presented at ISI equal or longer than $500 \mathrm{~ms}$. Error bars denote SEMs across participants.

\subsection{Experimental paradigm}

Stimuli consisted of brief pure tones ( $5 \mathrm{~ms}$ long, frequency $1000 \mathrm{~Hz}$ or $1200 \mathrm{~Hz}, \sim 70 \mathrm{~dB}$ SPL) presented binaurally via MEG-compatible air tubes. Stimulus delivery was controlled using Presentation software (Neurobehavioral Systems).

In each trial (see Figure 1A for an outline of the experimental paradigm; for more details see: Todorovic, et al., 2015), a central fixation cross was presented for 2-4 s, followed by a standard tone $(1000 \mathrm{~Hz})$ presented twice, with one of five inter-stimulus intervals (ISI) between the two tones $(250,375,500,625$, or $750 \mathrm{~ms})$. After the offset of the second tone, the fixation cross remained on the screen for $0.5-1 \mathrm{~s}$ and was subsequently replaced by a blank screen for 1.5-2 s. Occasionally, standard tones were replaced by deviant tones $(1200 \mathrm{~Hz} ; 9 \%$ trials) and participants were instructed to press a button with the right index finger as soon as they heard

\footnotetext{
foreperiod after the first tone, the more likely the presentation of the second tone), we also
} 
155

156

157

158

159

160

161

162

163

164

165

166

167

168

169

170

171

172

173

174

175

176

177

178

179

180

181

182

183

184

185

varied the width of the distribution of second tone presentation at each ISI. In blocks with focal temporal expectation, the second tone was played following an ISI of $500 \mathrm{~ms}$ in $70 \%$ trials, and following other ISIs in $7.5 \%$ trials each. As a result, participants could build up a narrow expectation of the second tone being presented $500 \mathrm{~ms}$ after the first tone. Conversely, in blocks with distributed temporal expectation, the second tone was played following an ISI of $500 \mathrm{~ms}$ in $24 \%$ trials, and following other ISIs in $19 \%$ trials each. In these blocks, participants could expect the second tone to be presented at any ISI with roughly the same probability. In total, four blocks with distributed temporal expectation (corresponding to 88 tones at the ISI $=500$ $\mathrm{ms}$ and 252 tones at the remaining ISIs), and two blocks with focal temporal expectation (corresponding to 120 tones at ISI $=500 \mathrm{~ms}$ and 52 tones at the remaining ISIs) were administered in each participant. The order of blocks was counterbalanced across subjects. Finally, the experimental paradigm included an orthogonal manipulation of temporal attention, whereby participants were instructed to attend to the first or second tone and press a button following a deviant tone presented in the attended position.

\subsection{Behavioural analyses}

We log-transformed the single-trial deviant detection RTs to normalise RT distribution. Trials with RTs outside of the mean $\pm 2 * S D$ range were excluded from analysis. Individual participants' mean RTs to deviants presented in the second position, calculated separately for ISIs shorter than, equal to, and longer than $500 \mathrm{~ms}$ respectively, were entered into a repeatedmeasures ANOVA with a 3-level factor ISI. For plotting purposes, data were back-transformed by exponentiating the individual mean RTs.

\subsection{MEG acquisition}

Magnetic fields induced by neural activity were measured using a whole-head MEG (VSM/CTF Systems) with 275 axial gradiometers. To enable continuous head localization, coils were placed at the nasion and right and left preauricular points, and monitored continuously during the experiment. Electro-oculogram (EOG) and electrocardiogram (ECG) were recorded to guide eye blink and heart beat artefact rejection, using 10-mm-diameter Ag$\mathrm{AgCl}$ surface electrodes.

\subsection{Event-related field analysis}


190 Continuous data were downsampled from $1200 \mathrm{~Hz}$ to $300 \mathrm{~Hz}$, notch-filtered at $50 \mathrm{~Hz}$ and highpass filtered at $0.1 \mathrm{~Hz}$ using a two-pass Butterworth filter. Artefacts induced by eye blinks were removed by subtracting two principal spatiotemporal modes associated with eye blinks (Ille, et al. 2002). Corrected sensor data were low-pass filtered at $48 \mathrm{~Hz}$.

Our aim was to compare second tones, that followed the first tone with varying ISIs, to each other. Given that tones at shorter ISIs arrived while the first tone was still being processed, we needed to assess what the evoked field to the second tone at all ISIs would look like in the absence of the first tone. To this end, rather than epoching the data, we modelled the evoked responses using convolution modelling in continuous sensor data estimated for the entire session using several regressors. Convolution modelling (Figure 2; cf. Litvak, et al., 2013; see Spitzer, et al., 2015; Auksztulewicz, et al., 2017 for applications) is equivalent to testing for the effects of independent stimulus events at each point in peristimulus time but at the same time allows for overlapping responses to successive trials, in the same way one would model fMRI time-series. Our principal aim was to estimate the evoked responses to tones presented at different ISIs relative to the first tone. To increase the number of events in each regressor, we pooled over ISIs shorter than $500 \mathrm{~ms}$ and longer than $500 \mathrm{~ms}$ respectively, resulting in three groups of ISIs (short, middle, long). Thus, the regressors included experimental regressors coding for tone onsets (standard tone in the first position; deviant tone in the first position; standard tone in the second position at ISI lower than, equal, and higher than $500 \mathrm{~ms}$ respectively; deviant tone in the second position pooled over all ISIs) and motor responses (button presses) as well as nuisance regressors. Tone regressors were further convolved with a binary regressor coding for temporal attention (on or off the tone of interest). Nuisance regressors included EOG time-series and its temporal derivative, as well as the ECG timeseries, to further reduce artefacts associated with eye movements and heart blinks. Furthermore, based on continuous head position measurement inside the MEG scanner, we calculated 6 movement parameters ( 3 translations and 3 rotations; cf. Stolk, et al., 2013), which were used as further nuisance regressors. 


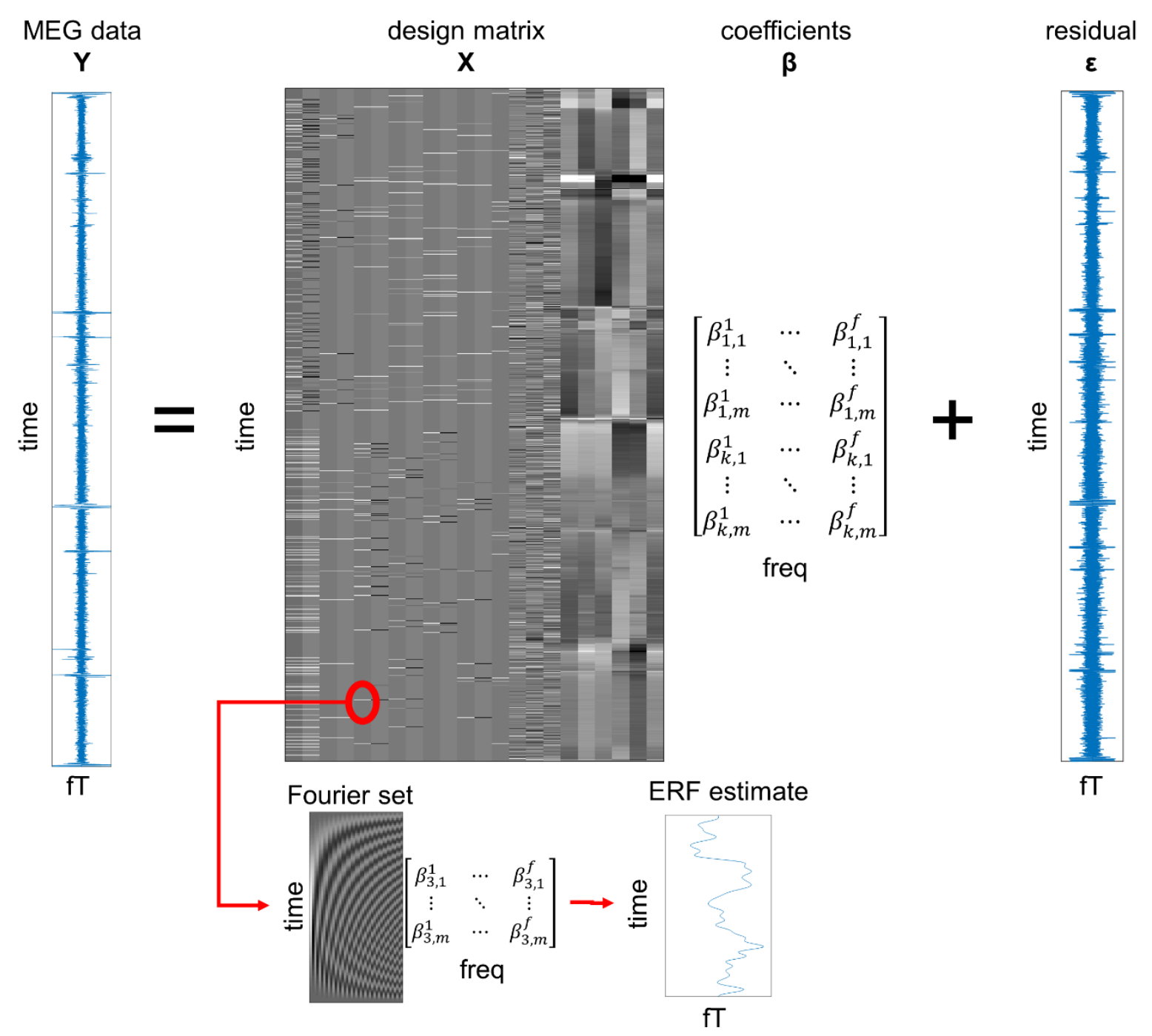

Figure 2. Convolution modelling. Individual participants' MEG data from the entire experiment (without epoching) were modelled in a general linear model. The design matrix specified the event and nuisance regressors (first 12 columns, left to right: standard onset at ISI $=0 \mathrm{~ms}$, deviant onset at ISI $=0$ $\mathrm{ms}$, standard onset at ISI $<500 \mathrm{~ms}$, standard onset at ISI $=500 \mathrm{~ms}$, standard onset at ISI $>500 \mathrm{~ms}$, deviant onset at ISI > $0 \mathrm{~ms}$; each with a corresponding modulation by attention on/off tone; further columns: button press, EOG and its temporal derivative, ECG, 6 motion regressors). Since each regressor was modelled as a Fourier time series (inset below), the ensuing Fourier coefficients can be summarised as a deconvolved ERF response to each event type and/or parametric regressor.

Single participants' evoked responses were modelled with a time range of $0 \mathrm{~ms}$ to $1000 \mathrm{~ms}$ relative to events of interest (tone onsets), and other events in the experimental session (button presses). While the actual window of interest encompassed the first $500 \mathrm{~ms}$ following tone onsets, we modelled evoked responses up to $1000 \mathrm{~ms}$ post-onset in order to deconvolve the

234 influence of neural responses to the first tone from the neural responses to the second tone, especially at short ISIs (shorter or equal to $500 \mathrm{~ms}$ ). Each event regressor was convolved with 
a 20-order Fourier basis set with a Hanning window, allowing for an estimation of evoked responses (specifically, their regressor coefficients) per MEG channel with the time-course estimates modulated up to $20 \mathrm{~Hz}$. The resulting spatiotemporal maps of regressor coefficients were converted into 3D images and entered into second-level general linear models (GLMs).

At a group level, we estimated the modulation of the tone-evoked neural responses by two different kinds of temporal expectation: passage of time (monotonically increasing with 243 increasing foreperiod) and tone onset distribution (following an inverted U-shape, approximating the average contextual expectation across the focal and distributed blocks;

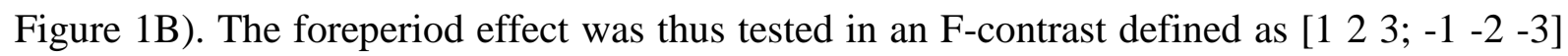
over the design matrix columns coding for tones presented in the second position at early, middle, and late ISIs respectively. Conversely, the contextual effect F-contrast was defined as [-1 $2-1 ; 1-2$ 1] over the same columns. Significant effects were inferred under the assumptions of random field theory (Kilner, et al. 2005) after thresholding the 3D (time x 2D topography) statistical parametric maps at $P<0.001$ (peak-level, uncorrected) and correcting $P$-values for multiple comparisons based on cluster size at a family-wise error rate at $P<0.05$.

\subsection{Source reconstruction}

We identified significant effects of foreperiod and context for two separate clusters (250-500 $\mathrm{ms}$, corresponding to the effect of foreperiod; and 425-500 ms, corresponding to the effect of context; see Results 3.2). We then based source reconstruction on a multiple sparse priors algorithm (Friston, et al. 2008) performed on these two time windows. For each time window, the estimated sensor-level evoked responses were first used to calculate contrast time-series, corresponding to the linear approximation to the passage-of-time and the inverted U-shaped approximation to the distribution effect. The resulting contrast time-series were treated as evoked responses whose underlying sources were inferred per participant using the multiple sparse priors source reconstruction. The single-participant 3D source activity maps were entered into a group-level GLM to infer those sources which are differentially sensitive to the temporal expectations based on foreperiod and distribution. Thus, significant effects were inferred per time window using a paired F-test, contrasting the source estimates corresponding to the passage-of-time effect and the distribution effect, after thresholding the $3 \mathrm{D}$ (spatial) 
multiple comparisons based on cluster size at a family-wise error rate at $P<0.05$. Sources were assigned anatomical labels using the SPM12 atlas provided by Neuromorphometrics, Inc.

A

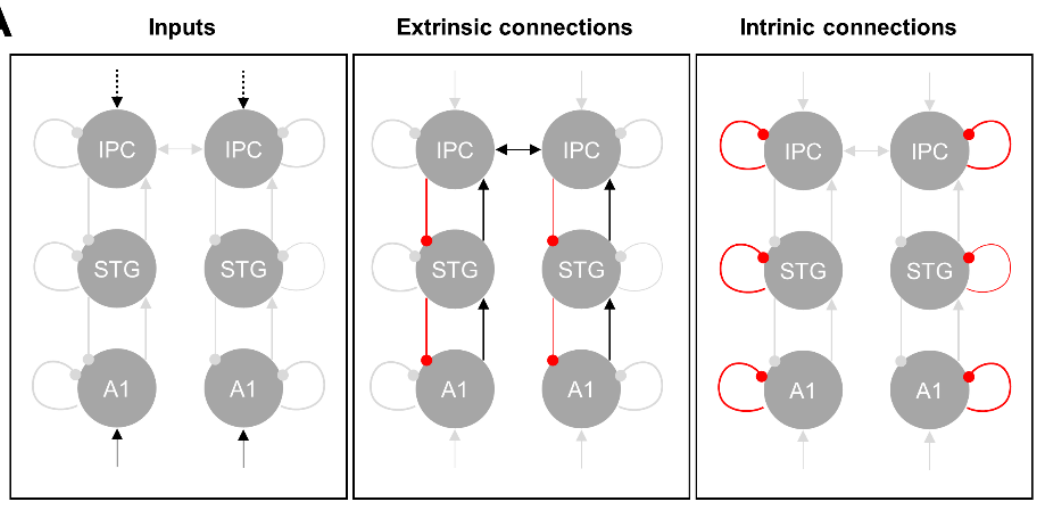

Inputs to both $\mathrm{A} 1$ and IPC? Intrinsic modulation by passage?
Extrinsic modulation by passage? Intrinsic modulation by context? Extrinsic modulation by context?

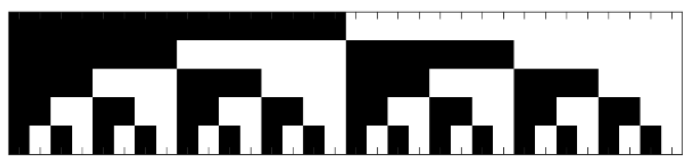

B
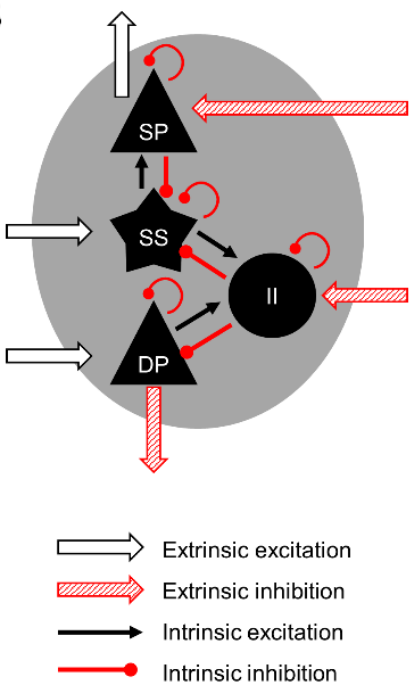

Figure 3. Dynamic causal modelling. (A) Model space: models had the same underlying architecture comprising 6 areas interconnected in an anatomically plausible way. The models differed with respects to which areas received external inputs (A1 only or A1 and IPC), and which connections (extrinsic vs. intrinsic) were modulated by each of the experimental manipulations (foreperiod vs. contextual expectation). Combinations of intrinsic modulations at the level of single regions are grouped for simplicity. This model space is depicted in the lower panel, with black/white shading representing absence/presence of a given input or modulation. (B) Each area corresponds to a microcircuit with 4 neural populations (SP: superficial pyramidal cells; DP: deep pyramidal cells; SS: spiny stellate cells; II: inhibitory interneurons) whose interactions determine the activity dynamics in the modelled network.

\subsection{Dynamic causal modelling}

To explain the observed effects of temporal expectation related to foreperiod and tone onset distribution on the evoked neural responses in a biophysically realistic model, we used dynamic causal modelling (DCM) in a neural mass model based on a canonical microcircuit (Figure 3B; cf. Pinotsis, et al. 2013). Specifically, the observed effects were modelled as changes in effective connectivity between and within nodes of a network comprised of several cortical sources. In DCMs based on a canonical microcircuit architecture, the activity in each source is modelled using ordinary differential equations which describe the changes in postsynaptic voltage and current in four neural populations. These populations (spiny stellate cells in the granular layer, supragranular/superficial and infragranular/deep pyramidal cells, and inhibitory 
interneurons) are characterised by distinct profiles of ascending and descending connectivity both extrinsically (linking different sources) and intrinsically (coupling neural populations within a source). Specifically, there is a laminar asymmetry in the outputs of each source — for instance, superficial pyramidal cells propagate ascending signals to hierarchically higher areas, whereas deep pyramidal cells propagate descending signals to hierarchically lower areas. The output of each source is further modulated by intrinsic connectivity parameters, describing the strength of the self-inhibition of each neural population. The equations describing the dynamics at each source are provided below:

1. $\dot{V}_{S S}=I_{S S}$

2. $\dot{I}_{S S}=\kappa_{S S}\left(A^{F} \sigma\left(V_{S P}\right)-\gamma_{S S \rightarrow S S} \sigma\left(V_{S S}\right)-\gamma_{S P \rightarrow S S} \sigma\left(V_{S P}\right)-\gamma_{I I \rightarrow S S} \sigma\left(V_{I I}\right) E(x)\right)-2 \kappa_{S S} V_{S S}-\kappa_{S S}^{2} I_{S S}$

3. $\quad \dot{V}_{I I}=I_{I I}$

4. $\quad \dot{I}_{I I}=\kappa_{I I}\left(-A^{B} \sigma\left(V_{D P}\right)+\gamma_{S S \rightarrow I I} \sigma\left(V_{S S}\right)+\gamma_{D P \rightarrow I I} \sigma\left(V_{D P}\right)-\gamma_{I I \rightarrow I I} \sigma\left(V_{I I}\right)\right)-2 \kappa_{I I} V_{I I}-\kappa_{I I}^{2} I_{I I}$

5. $\quad \dot{V}_{S P}=I_{S P}$

6. $\dot{I}_{S P}=\kappa_{S P}\left(-A^{B} \sigma\left(V_{D P}\right)+\gamma_{S S \rightarrow S P} \sigma\left(V_{S S}\right)-\gamma_{S P \rightarrow S P} \sigma\left(V_{S P}\right)\right)-2 \kappa_{S P} V_{S P}-\kappa_{S P}^{2} I_{S P}$

7. $\dot{V}_{D P}=I_{D P}$

8. $\dot{I}_{D P}=\kappa_{D P}\left(A^{F} \sigma\left(V_{S P}\right)-\gamma_{D P \rightarrow D P} \sigma\left(V_{D P}\right)-\gamma_{I I \rightarrow D P} \sigma\left(V_{I I}\right)\right)-2 \kappa_{D P} V_{D P}-\kappa_{D P}^{2} I_{D P}$

The distinct neural populations comprising a canonical microcircuit are indicated by subscripts SS (spiny stellate cells), II (inhibitory interneurons), SP (superficial pyramidal cells), and DP (deep pyramidal cells). The voltage and current of a population $m$ are denoted by $V_{\mathrm{m}}$ and $I_{\mathrm{m}}$ respectively, determined by the synaptic rate constant $\kappa_{m} s$. The sigmoid operator $C$ transforms the postsynaptic potential into firing rate. The extrinsic ascending and descending connections are denoted by $A^{\mathrm{F}}$ and $A^{\mathrm{B}}$ respectively. Intrinsic connections from population $m$ to $n$ are denoted by $\gamma_{m \rightarrow n}$. Crucially, the intrinsic self-inhibition of superficial pyramidal cells $\gamma_{S P \rightarrow S P}$ determines the gain of ascending connections, which has been linked to the precision of prediction error signalling in the predictive coding framework (Feldman and Friston, 2010). Finally, thalamic input $u$ scaled by its weight $C$ contributes to the changes in current of spiny stellate cells at the lowest level of the hierarchy. This model has been used in several other DCM studies of evoked responses (e.g., Moran, et al., 2013; Auksztulewicz \& Friston, 2015; Brown \& Friston, 2012), and neurophysiological inference using DCM has been validated in animal models (Moran, et al., 2011) and invasive recordings in humans (Papadopoulou, et al., 2015). 
A model space containing two model families with 256 competing models each was designed to disambiguate alternative hypotheses regarding the connectivity patterns modulated by temporal expectation based on passage of time vs. distribution of stimulus events (Figure 3A). All models were based on the same basic network, comprising 3 bilateral sources identified in the source reconstruction of the observed effects (see Results 3.3), namely early auditory cortex (A1), superior temporal gyrus (STG), and inferior parietal cortex (IPC). The two families differed with respect to the subset of regions receiving driving (e.g., thalamic) input: in one family, the driving input was assumed to only reach bilateral A1 (cf. Auksztulewicz \& Friston, 2015; Moran, et al., 2013), while in the other family the driving input reached both A1 and IPC, akin to non-sensory input regions for expectation signals modelled in other studies using DCM connections to be modulated by either of the experimental factors (passage of time and/or context). Specifically, we considered models in which each of the experimental factors could independently modulate the following subsets of connections: (1) A1 gain; (2) STG gain; (3) IPC gain; (4) extrinsic connectivity between regions. In the group-level analysis, first - for computational feasibility - we fitted only the full models (i.e., allowing for all connections to be modulated by both experimental factors) to each participant's data. These models were fitted to the event-related fields in the $0-500 \mathrm{~ms}$ range relative to tone onset across all MEG channels, resulting in predicted sensor-level data (specifically, 8 principal spatiotemporal modes accounting for $>99 \%$ of the variance across all sensors and time points). The reduced models (allowing for only a subset of connections to be modulated) were not fitted individually, but instead their parameter estimates (describing the effective extrinsic and intrinsic connectivity parameters) and model evidence (reflecting the model fit while penalising for model complexity) were obtained per participant using Bayesian model reduction of the respective full models (Friston, et al., 2016). Following this step, we obtained model evidence and parameter estimates for each model and participant. Second, to ensure that group-level model evidence or parameter estimates are not driven by outliers or models with a poor fit, we used parametric empirical Bayes to obtain group-level parameter estimates assuming that singleparticipant parameter estimates for each model come from a normal distribution (Friston, et al., 2016). Thus, we obtained group-level model evidence and parameter estimates for each model. Finally, to accommodate uncertainty inherent in comparing such a large number of models, we used Bayesian model averaging (Friston, et al., 2016) of the group-level models in the winning model family, which integrates single-model parameter estimates weighting them by the evidence of each respective model. The average model contained parameter estimates (mean 
and covariance estimates) used for statistical inference. Parameters were considered significantly modulated if they were different from 0 at a posterior probability threshold of $95 \%$ (Bonferroni-corrected across parameters).

\section{RESULTS}

\subsection{Behavioural results}

Participants detected on average $95.6 \%$ of the deviants ( $\mathrm{SD}=5.3$ across participants). For deviants presented in the second position, the waiting time (ISI) had a significant effect on reaction times $\left(\mathrm{F}_{(2,46)}=9.29, \mathrm{p}<.001\right.$; see Figure $\left.1 \mathrm{C}\right)$, with deviant tones presented at shorter intervals after the first tone (ISI<500) being detected more slowly than deviants presented at the middle interval (ISI $=500 \mathrm{~ms} ; \mathrm{t}_{(23)}=3.33, \mathrm{p}=0.003$; mean RT at short ISIs: $561 \mathrm{~ms}$, medium ISIs: $499 \mathrm{~ms}$ ) or at longer intervals (ISI $>500, \mathrm{t}_{(23)}=4.97, \mathrm{p}<.001$; mean RT at long ISIs: 492 $\mathrm{ms})$. We found no evidence for a difference between RTs to deviants presented at medium vs. long ISIs $\left(\mathrm{t}_{(23)}=0.31, \mathrm{p}>.05\right)$. However, given the relatively low number of trials with responses to deviants per condition (14 trials on average), the presence or absence of behavioural effects on the deviant tones should not be interpreted as an indicator for the presence or absence of neural effects on the standard tones (see below, 3.2).

\subsection{Event-related fields - sensor space analysis}

Both the foreperiod until the tone onsets and the distribution of tone onset times had an influence on sensor-level ERF amplitude. (Figure 4A). Specifically, the passage of time until tone onset (foreperiod) significantly modulated ERF amplitude between 267-500 ms with a bilateral topography. The distribution of tone onsets, on the other hand, had a significant effect on ERF amplitude later in time (between $427-500 \mathrm{~ms}$ ) and with a left-lateralised topography. We next wanted to know whether these clusters can be uniquely linked to foreperiod and temporal distribution expectations respectively, or, conversely, if they represent a mixture of both effects. To this end we repeated the analysis by (1) testing the effect of foreperiod while masking out the effect of onset distribution (masking threshold $\mathrm{p}<.001$, uncorrected) and inferring the significant effects at $\mathrm{p}<.05$, FWE-corrected; (2) testing the effect of onset distribution while masking out the effect of foreperiod (using identical thresholds); (3) testing the conjunction of foreperiod and distribution effects (thresholded at $\mathrm{p}<.001$ and corrected for 
multiple comparisons at $\left.\mathrm{p}_{\mathrm{FWE}}<.05\right)$. Here we found that the effects of foreperiod and context were independent of each other (Figure 4B). There was no evidence of overlap in the two clusters, as identified in the conjunction analysis (no conjunction cluster significant at a threshold of $\mathrm{p}<.001$, after correcting for multiple comparisons at $\left.\mathrm{p}_{\mathrm{FWE}}<.05\right)$.
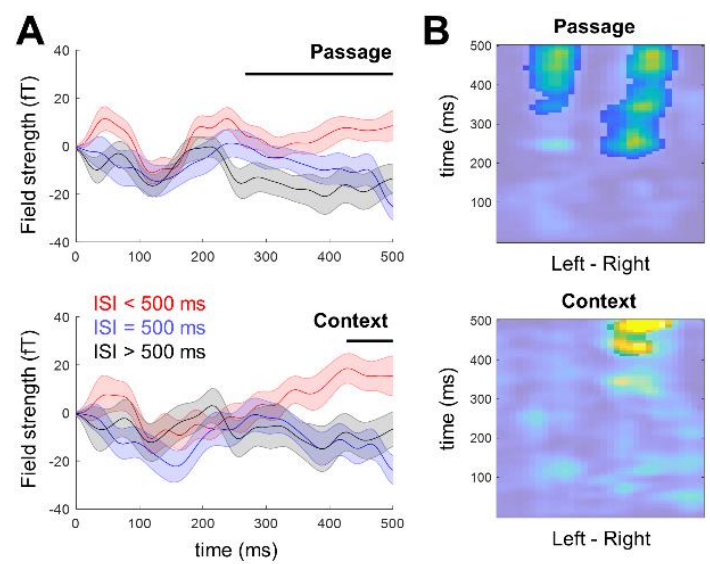

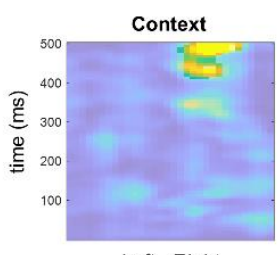

Left - Right
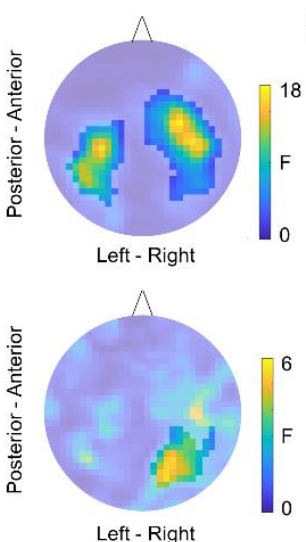

C
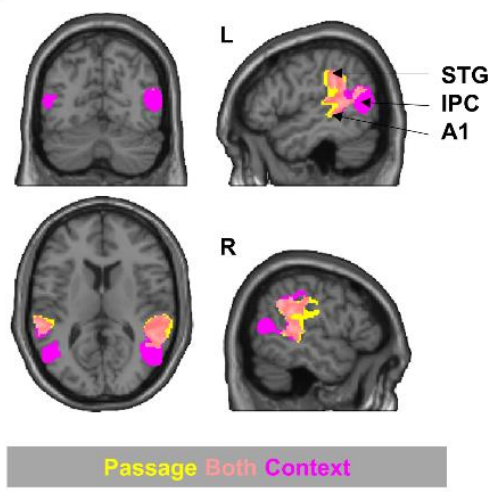

Figure 4. Differential effects of foreperiod and contextual expectation on evoked MEG responses.

(A) Time-courses of averaged MEG amplitude extracted from channels showing significant effects of foreperiod (upper) and context (lower). Shaded areas denote SEM over participants. Black bar denotes time window of a significant cluster also shown in (B). (B) Both columns show maps of F statistics over parametric effects of passage (upper) and context (lower). Left column shows the time course of the effect on the Y axis. Right column shows the topography of the effect. The effect of foreperiod starts earlier than the effect of context. When thresholding at $\mathrm{p}<.001$ and correcting using $\mathrm{pFWE}<.05$, foreperiod has significant bilateral effects between $267-500 \mathrm{~ms}$ while context has a left-lateralised effect between $427-500 \mathrm{~ms}$ (unmasked areas). (C) Source reconstruction. In yellow, the significant ( $\mathrm{p}<.001$, pFWE<.05) difference between sources underlying passage and context in the long time window. In magenta, the significant $(\mathrm{p}<.001, \mathrm{pFWE}<.05)$ difference between sources underlying passage and context in the short late time window. In orange, the overlap between the two. Slices centred at $[ \pm 52-$ 65 11] MNI coordinates. A1: primary auditory cortex; STG: superior temporal gyrus; IPC: inferior parietal cortex.

\subsection{Dynamic causal modelling - source space analysis}

Our next step was to reconstruct the sources of the sensor-level parametric effects of foreperiod and tone distribution expectation (Figure 4C; Table 1). This analysis revealed that for a long time window (250-500 ms), in which the effect of foreperiod modulated ERF amplitude, the power of source-level activity in bilateral early auditory cortices (A1) and superior temporal gyri (STG) significantly differed between temporal expectations stemming from foreperiod and 
422

423

424

425

426

427

428

429

430

431

432

433

434

435

436

437

438

439

440

441

442

443

444

445

446

447

448

tone onset distribution (voxels thresholded at $\mathrm{p}<.001$ and corrected using $\mathrm{pFWE}<.05$ ). Apart from these two bilateral regions, an analysis of the shorter, later time window (425-500 ms) in which ERF amplitude was modulated by expectation of tone distribution, revealed that bilateral sources including A1, STG, and additionally inferior parietal cortex (IPC) once again significantly differed between the two kinds of temporal expectation. In sum, IPC was uniquely sensitive to expectations of distribution of events in time, while A1 and STG were sensitive to both this distribution and the passage of time. We next used these 3 bilateral regions as a basis for subsequent dynamic causal modelling. The full models (with all connections allowed to be modulated by both kinds of temporal expectations) explained on average $50.64 \%$ (SD = $15.71 \%$ ) variance of the individual-participant MEG channel $x$ time $x$ condition data. Figure 5B shows the average observed (measured) and predicted (modelled) data per channel and time point.
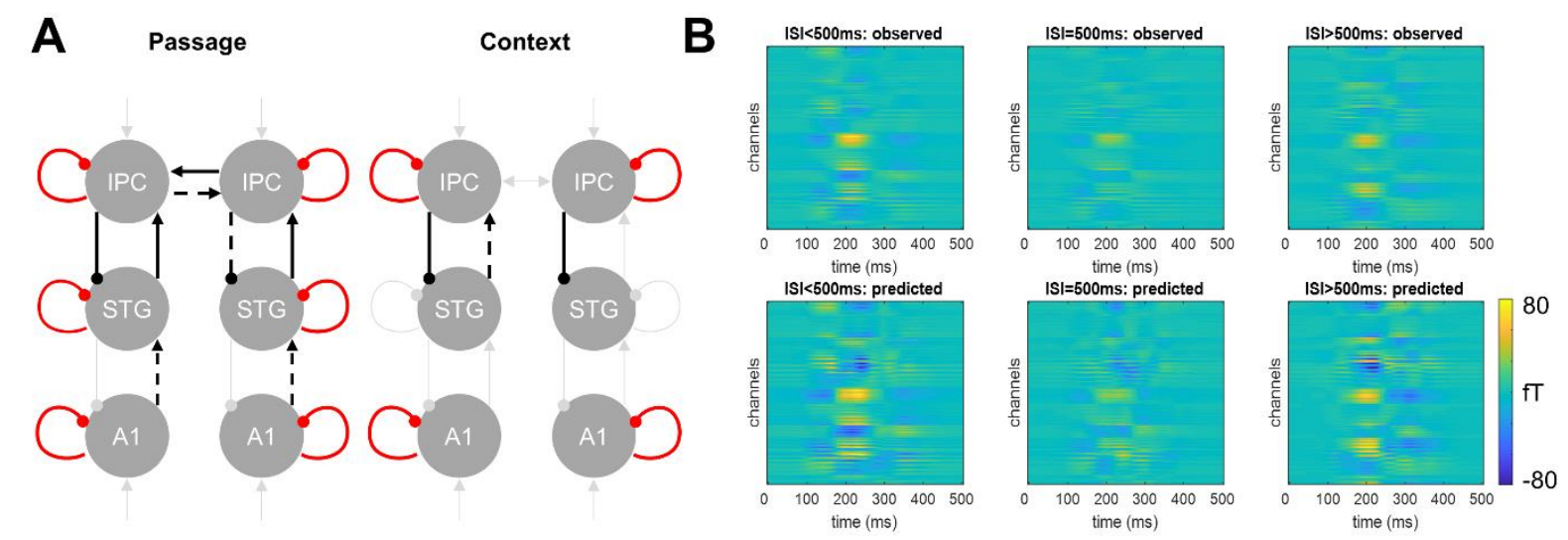

Figure 5. Dynamic causal modelling results. (A) Modulatory parameters of the winning model for the effects of passage of time (foreperiod; left) and context (right). Black: excitatory, Red: inhibitory, solid: stronger, dashed: weaker; light grey: connections not modulated by a given experimental factor. (B) Observed (upper row) and predicted (lower row) responses for each modelled condition (columns), time point ( $\mathrm{X}$ axis), and MEG channel ( $\mathrm{Y}$ axis).

Our model space included two families of models: in one family, inputs were assumed to only reach bilateral A1, representing ascending sensory information (cf. Auksztulewicz \& Friston, 2015; Moran, et al., 2013), while in the other family the driving input reached both A1 and IPC. This second family was based on DCM literature suggesting that expectation signals are effectively modelled as inputs to non-sensory regions (Philips, et al., 2016; Gilbert \& Moran, 2016), and indeed this family had higher model evidence on average in our dataset (posterior family probability $>99.9 \%$ ). In each family, the associated models differed with respect to the 
pattern of possible of extrinsic and intrinsic connections which would be modulated by

450 foreperiod and/or temporal distribution. The Bayesian model average of all models in the

451 winning family indicated that, while both passage-of-time and temporal distribution modulated

452 the weight of extrinsic connections (linking different regions) and intrinsic connections

453 (modelling gain within regions), their effects were dissociable at the level of specific

454 connections both within and across regions. The posterior parameters of the winning model are

455 plotted in Figure 5A and summarised in Table 2. These parameters indicate that, with stronger

456 predictions of passage of time, the lower-order ascending (excitatory) connections between A1

457 and STG became weaker, while the higher-order ascending connections between STG and IPC

458 became stronger. The descending (inhibitory) connections were modulated by passage of time

459 only between higher-order regions (IPC and STG). The effects of passage of time on these

460 descending connections were asymmetric, showing stronger inhibition in the left hemisphere

461 and weaker inhibition in the right hemisphere. These effects were accompanied by an

462 asymmetric modulation of lateral (cross-hemispheric) connections between left and right IPC,

463 with net effective connectivity shifted towards the left hemisphere. The effects of expectation

464 due to temporal distribution on extrinsic connections were limited to higher-order connectivity

465 between STG and IPC, showing a left-lateralised decrease of ascending excitation and a

466 bilateral increase in descending inhibition.

467

468 In addition to the dissociable effects on extrinsic connections, expectation due to foreperiod 469 and temporal distribution also showed dissociable effects on intrinsic connections. Specifically, 470 while increasing expectation due to foreperiod increased intrinsic connectivity in all regions 471 bilaterally, expectation due to temporal distribution increased intrinsic connectivity in bilateral 472 A1 and IPC but not in STG.

\section{DISCUSSION}

477 Attending to events in time has been linked to a broad network of areas, reflecting the complexity of temporal expectation (Coull, et al. 2004; Muller and Nobre 2014). Here we investigated whether passage of time and tracking the distribution of events in time have dissociable neural substrates. We employed a two-tone paradigm where onsets of the second tone were normally distributed along five inter-stimulus intervals, and we looked at how the second tone in these pairs was processed as a function of elapsed time, and of the likelihood of 
483

484

485

486

487

488

489

490

491

492

493

494

495

496

497

498

499

500

501

502

503

504

505

506

507

508

509

510

511

512

513

514

515

516

the tone arriving at that particular inter-stimulus interval. We modelled passage of time and temporal distribution separately, with passage of time corresponding to a model where evoked activity reduced as the inter-stimulus intervals got longer (i.e. predictability grew), and distribution of events in time corresponding to a model where evoked activity was minimal at the middle interval (where predictability was greatest) but grew towards the edges of the distribution. We found that these two types of temporal expectation correlated with activity in a similar set of areas, but that the modulations began at different moments in time, and that they affected network connectivity in distinct ways.

Behaviourally, we found that tones that arrived before the most frequent, middle ISI, were paired with slower response times, confirming that likelihood had an effect on how tones were processed. A recent study with normally distributed tone onsets also found faster responses to tones with more predictive foreperiods (Herbst, Fiedler, and Obleser 2018), which echoes previous findings (Hsu, Hämäläinen, and Waszak 2013; Coull and Nobre 1998; Miniussi, et al. 1999). On the neural level, since the tones were presented quickly one after the other (with the ISIs ranging from $250 \mathrm{~ms}$ to $750 \mathrm{~ms}$ ), we first applied convolution modelling to estimate the evoked field to the second tone while controlling for the influence of the first tone. After this step, we were able to include early, middle and late arriving tones into a single search for cortical sources. We found two clusters of activity, one beginning early (starting at $267 \mathrm{~ms}$ after stimulus onset) and corresponding to passage of time, and one beginning late (starting at 427 ms after stimulus onset) and corresponding to temporal distribution of tone events. Importantly, we found that the neural effects related to these two types of temporal expectation were separable from each other.

We then localised the effects of temporal expectation to sources corresponding, bilaterally, to the primary auditory cortex (A1), superior temporal gyrus (STG) and inferior parietal cortex (IPC). While A1 and STG were sensitive to both this distribution and the passage of time (corresponding to the early and late clusters of activity in the ERF time-series analysis), IPC was uniquely sensitive to expectations of distribution of events in time (corresponding specifically to the late activity clusters). In previous research, A1 has been implicated not only as a hub for auditory processing, but also as a modality-independent timekeeper (Kanai, et al. 2011). The STG and IPC have, similarly, often been found to be modulated by temporal expectation (Morillon and Baillet 2017; Wiener, Turkeltaub, and Coslett 2010; Rao, Mayer, and Harrington 2001; Cui, et al. 2009; Coull, Nazarian, and Vidal 2008). One area that is also 
517 frequently found to be involved in time estimation, is the supplementary motor area (SMA)

518 (Herbst, Fiedler, and Obleser 2018; Bueti, et al., 2010; Coull, et al., 2008; Akkal, et al., 2004).

519 It has even been suggested that processing of time has motor origins (Morillon and Baillet

520 2017). Interestingly, we did not observe SMA activity to be involved in our temporal hazard.

521 Potentially this might be because the temporal processing in our study was implicit: although it

522 aided task performance, this was a pitch discrimination task, and not one that involved

523 estimating durations. However, one other study with a tone discrimination task involving

524 similarly distributed tone onset times also found SMA activity (Herbst, Fiedler, and Obleser

525 2018). In that study, SMA was involved in foreperiod tracking in blocks with flat (i.e.

526 equiprobable) onset time distributions but not in blocks with strong contextual expectations of

527 the distribution. It is therefore likely that our search for cortical sources, which was specifically

528 sensitive to areas involved both in passage of time and contextual distribution of events in time,

529 led to SMA being excluded.

530

531 We next looked at how each of these two types of temporal expectation modulated connectivity 532 within and between A1, STG and IPC. We found that both types of temporal expectation 533 affected the strength of connections between regions in the auditory network. We were 534 particularly interested in testing whether changes in connection weights would be in line with 535 predictive coding, namely, corresponding to decreases in feedforward excitation, increases in 536 feedback inhibition and increases in intrinsic inhibition when a tone is more predictable. 537 However, the changes in connectivity profiles that were affected by temporal expectation were 538 more varied, especially when it came to tracking predictability as a function of elapsed time.

540 The most prominent common feature between the two types of temporal expectation was how 541 they modulated intrinsic connections in bilateral A1, STG and IPC. With growing tone onset 542 probability, whether due to passage of time or due to their distribution, intrinsic connections 543 consistently led to greater inhibition of neural activity (i.e. lower gain). Gain modulation has 544 indeed been posited as a core feature of predictive processing (Garrido, et al., 2009; 545 Auksztulewicz \& Friston, 2016). In the context of repetition suppression, when an initially 546 novel (unpredicted) stimulus starts repeating and forming a standard (predicted) stimulus, 547 neural gain - modelled as intrinsic connections - shows a relative decrease following the first 548 repetitions (when a prediction is being established) and a gradual rebound with later repetitions 549 (when a prediction is fully formed; Garrido, et al., 2009). A similar change in intrinsic 550 connectivity was evident here in the entire network for predictability related to elapsed time, 
551 and in A1 and IPC, but not STG, for predictability associated with temporal distribution. A

552 number of previous studies have indicated a role for the STG in temporal processing. For 553 instance, BOLD activity evoked by target onset in both STG and SMA has been shown to reflect 554 the cumulative hazard of a target appearing at a given moment, independent of modality 555 (auditory vs. visual) or the presence of a motor response (Cui, et al., 2009). Importantly, when 556 participants were informed of the exact target onset in that study, BOLD signal no longer 557 correlated with the foreperiod, suggesting that activity in these regions did not simply reflect 558 passage of time, but rather a temporal probability estimate. Another study found that STG (but 559 not A1 or IPC) was involved in estimating relative stimulus duration (Coull, et al., 2008). 560 Therefore, a plausible explanation for the difference in STG gain modulation between the two 561 types of temporal expectation is that a more involved time estimation was necessary for 562 estimating the expected peaks of temporal distribution in our study, which required activity in 563 STG, preventing self-inhibition.

565 The added complexity of assessing temporal distribution (relative to passage of time) was also visible in the fact that predictability due to distribution was largely tracked in higher-level connections between IPC and STG. Differences in prediction complexity have previously been linked to hierarchical differences in prediction and prediction error signalling, with hierarchically higher areas integrating information over longer time scales and representing more complex predictions (Auksztulewicz \& Friston, 2016; Chao, et al., 2018; Kiebel, et al. 2008). In the current study, fully in line with predictive coding, tones that were more predictable

572 based on the distribution (i.e. middle ISI tones relative to both early and late tones) led to less 573 feedforward activation from STG to IPC, and more feedback inhibition from IPC to STG.

575 Similarly, if the prediction came from monitoring elapsed time, less excitation was fed forward 576 from A1 to STG. The same study that found reduced gain during prediction formation also 577 found a monotonically decreasing feedforward excitation from A1 to STG, as well as a 578 reduction in ERPs, in repeated standard tones (Garrido, et al. 2009). Although there was no 579 manipulation of stimulus timing, the similarity with our experiment lies in stimulus 580 predictability: standard tones are predictable and expected, especially when repeated multiple 581 times, and therefore less forward excitation likely mediates reduced prediction error signalling 582 at lower levels of the cortical hierarchy (Auksztulewicz \& Friston, 2016). 
584 However, at higher levels of the cortical hierarchy, the changes in connectivity begin to diverge 585 from what predictive coding models would posit. Firstly, more (not less) excitation was fed 586 forward from bilateral STG to IPC after the occurrence of a more predictable tone. Secondly, 587 when it comes to feeding inhibition back from IPC to STG, passage of time led to an opposite 588 effect in the two hemispheres, with inhibition increasing with stronger predictions in the left 589 hemisphere, but decreasing in the right hemisphere, amounting to stronger net activation in the 590 right IPC and weaker activation in the left hemisphere. Thirdly, stronger predictability with 591 passage of time led to an asymmetric modulation of cross-hemispheric connectivity between 592 the left and right IPC, amounting to the right IPC exerting stronger influence on the left IPC 593 than vice versa. While stimulus expectation is most often linked to a reduction in forward 594 signalling, expectation enhancement has also occasionally been observed (Segaert et al., 2013; 595 Recasens et al., 2014) and the underlying mechanisms are still under debate (Auksztulewicz \& 596 Friston, 2015). For instance, the net effect of expectation on neural activity may be mediated 597 by non-specific response dampening or by selective response sharpening (de Lange, et al., 598 2018); furthermore, expectation of different features may be mediated by qualitatively different 599 cortical circuits (Auksztulewicz, et al., 2017). Interestingly, the reversing of prediction-related inhibition of neural activity by other factors such as attention has been described before (Kok, et al., 2012). In the current study, lower-level inhibition of ascending signalling may reflect a non-specific dampening of prediction error signalling as passage of time increases the predictability of a received stimulus; higher-level ascending excitation, on the other hand, may reflect a signal halting the ongoing time estimation as the stimulus has been received and can

605 be processed by other regions, including the attentional network. A right-hemispheric IPC 606 asymmetry has previously been reported specifically in a paradigm based on encoding time intervals (Rao, Mayer, and Harrington 2001) and linked to the lateralisation of the frontoparietal attentional network.

The differences between how two types of temporal expectation modulated connectivity suggest that, while stronger predictions can lead to less feedforward excitation and more feedback inhibition, these changes are not passed along the entire cortical hierarchy. In fact, they are more likely to be contained between a smaller number of cortical areas that are directly specific to a certain type of information processing, with, in this case, the more complex predictability related to tone onset distribution, modulating only higher order connections but not influenced by changes in activation coming from the primary auditory cortex. In addition, at the higher levels of the hierarchy the prediction errors were not suppressed by prediction 
618 related to passage of time, but instead changed the connectivity pattern in a different, often

619 opposite way. Taken together, our results show demonstrate that different aspects of temporal

620 expectation can be dissociable in terms of the latency of neural responses, the underlying

621 sources and connectivity patterns, leading to a more nuanced view of how prediction and

622 prediction error signalling may be expressed in cortical circuits.

623

624

625 
TABLES

627

\begin{tabular}{|c|c|c|c|c|c|c|}
\hline $\begin{array}{l}\text { Time } \\
\text { window }\end{array}$ & $\begin{array}{l}\text { Cluster- } \\
\text { level } p_{F W E}\end{array}$ & $\begin{array}{l}\text { No. } \\
\text { voxels }\end{array}$ & $\begin{array}{l}\text { Peak- } \\
\text { level F }\end{array}$ & $\begin{array}{l}\text { Peak- } \\
\text { level Z }\end{array}$ & $\begin{array}{l}\text { MNI } \\
\text { coordinates }\end{array}$ & Anatomical labels \\
\hline \multirow{6}{*}{$\begin{array}{l}250-500 \\
\mathrm{~ms}\end{array}$} & \multirow[t]{3}{*}{0.009} & \multirow[t]{3}{*}{2341} & 40.05 & 4.63 & $56-4016$ & rSTG \\
\hline & & & 35.39 & 4.44 & $48-3814$ & rSTG / rPT \\
\hline & & & 32.49 & 4.30 & $54-2812$ & rPT \\
\hline & \multirow[t]{3}{*}{0.010} & \multirow[t]{3}{*}{2304} & 37.30 & 4.52 & $-60-3216$ & IPT \\
\hline & & & 36.20 & 4.47 & $-56-3214$ & IPT \\
\hline & & & 18.66 & 3.48 & $-54-426$ & ISTG \\
\hline \multirow{6}{*}{$\begin{array}{l}425-500 \\
\mathrm{~ms}\end{array}$} & \multirow[t]{3}{*}{0.014} & \multirow[t]{3}{*}{2501} & 33.21 & 4.34 & $60-4418$ & rSTG \\
\hline & & & 19.20 & 3.52 & $56-2812$ & rPT \\
\hline & & & 18.50 & 3.46 & $44-646$ & $\begin{array}{l}\text { rIPC } \\
\text { (Ang/MTG/MOG) }\end{array}$ \\
\hline & \multirow[t]{3}{*}{0.022} & \multirow[t]{3}{*}{2205} & 30.10 & 4.19 & $-58-3414$ & IPT \\
\hline & & & 20.35 & 3.60 & $-48-6612$ & $\begin{array}{l}\text { IIPC } \\
\text { (Ang/MTG/MOG) }\end{array}$ \\
\hline & & & 19.85 & 3.57 & $-54-426$ & ISTG \\
\hline
\end{tabular}

629 Table 1. Source reconstruction results. STG: superior temporal gyrus; PT: planum temporale;

630 IPC: inferior parietal cortex; Ang: angular gyrus; MTG: medial temporal gyrus; MOG: medial 631 occipital gyrus.

\begin{tabular}{|c|c|c|c|c|c|}
\hline $\begin{array}{l}\text { Modulatory } \\
\text { effect }\end{array}$ & Parameter & $\begin{array}{l}\text { Posterior } \\
\text { mean }\end{array}$ & $\begin{array}{l}\text { Posterior } \\
\text { probability }\end{array}$ & Description & Net effect \\
\hline \multirow[t]{13}{*}{ Foreperiod } & $\mathrm{B}(1,1)$ & .3239 & $>.9999$ & $\begin{array}{ll}\text { A1 } & \text { self-inhibition } \\
\text { (left) }\end{array}$ & Inhibitory \\
\hline & $\mathrm{B}(3,1)$ & -.6631 & $>.9999$ & $\begin{array}{l}\text { A1-STG connection } \\
\text { (left) }\end{array}$ & Inhibitory \\
\hline & $\mathrm{B}(2,2)$ & .1038 & $>.9999$ & $\begin{array}{l}\text { A1 self-inhibition } \\
\text { (right) }\end{array}$ & Inhibitory \\
\hline & $\mathrm{B}(4,2)$ & $\begin{array}{l}.2707 \\
\end{array}$ & $>.9999$ & $\begin{array}{l}\text { A1-STG connection } \\
\text { (right) }\end{array}$ & Inhibitory \\
\hline & $\mathrm{B}(3,3)$ & 0.2587 & $>.9999$ & $\begin{array}{l}\text { STG self-inhibition } \\
\text { (left) }\end{array}$ & Inhibitory \\
\hline & $\mathrm{B}(5,3)$ & 0.4014 & $>.9999$ & $\begin{array}{l}\text { STG-IPC connection } \\
\text { (left) }\end{array}$ & Excitatory \\
\hline & $\mathrm{B}(6,4)$ & 0.1655 & 0.9993 & $\begin{array}{l}\text { STG-IPC connection } \\
\text { (right) }\end{array}$ & Excitatory \\
\hline & $\mathrm{B}(3,5)$ & -.1621 & $>.9999$ & $\begin{array}{l}\text { IPC-STG connection } \\
\text { (left) }\end{array}$ & Disinhibitory \\
\hline & $\mathrm{B}(5,5)$ & .0960 & .9998 & $\begin{array}{l}\text { IPC self-inhibition } \\
\text { (left) }\end{array}$ & Inhibitory \\
\hline & $\mathrm{B}(6,5)$ & .2214 & .9999 & $\begin{array}{lr}\begin{array}{l}\text { IPC } \\
\text { hemispheric }\end{array} & \begin{array}{r}\text { cross- } \\
\text { (left- } \\
\text { right) }\end{array} \\
\end{array}$ & Excitatory \\
\hline & $\mathrm{B}(4,6)$ & .2141 & $>.9999$ & $\begin{array}{l}\text { IPC-STG connection } \\
\text { (right) }\end{array}$ & Inhibitory \\
\hline & $\mathrm{B}(5,6)$ & -.3083 & $>.9999$ & $\begin{array}{ll}\text { IPC } & \text { cross- } \\
\text { hemispheric } & \text { (right- } \\
\text { left) } & \end{array}$ & Inhibitory \\
\hline & $\mathrm{B}(6,6)$ & .0832 & .9996 & $\begin{array}{l}\text { IPC } \\
\text { (right) }\end{array}$ & Inhibitory \\
\hline
\end{tabular}


bioRxiv preprint doi: https://doi.org/10.1101/769372; this version posted September 14, 2019. The copyright holder for this preprint (which was not certified by peer review) is the author/funder, who has granted bioRxiv a license to display the preprint in perpetuity. It is made available under aCC-BY 4.0 International license.

633

\begin{tabular}{|c|c|c|c|c|c|}
\hline \multirow[t]{7}{*}{ Context } & $\mathrm{B}(1,1)$ & .1586 & $>.9999$ & $\begin{array}{ll}\text { A1 } & \text { self-inhibition } \\
\text { (left) }\end{array}$ & Inhibitory \\
\hline & $\mathrm{B}(2,2)$ & .6086 & $>.9999$ & $\begin{array}{l}\text { A1 self-inhibition } \\
\text { (right) }\end{array}$ & Inhibitory \\
\hline & $\mathrm{B}(6,4)$ & -.1775 & .9999 & $\begin{array}{l}\text { STG-IPC connection } \\
\text { (right) }\end{array}$ & Inhibitory \\
\hline & $\mathrm{B}(3,5)$ & .0888 & .9992 & $\begin{array}{l}\text { IPC-STG connection } \\
\text { (left) }\end{array}$ & Inhibitory \\
\hline & $\mathrm{B}(5,5)$ & .2643 & $>.9999$ & $\begin{array}{l}\text { IPC self-inhibition } \\
\text { (left) }\end{array}$ & Inhibitory \\
\hline & $\mathrm{B}(4,6)$ & .2718 & $>.9999$ & $\begin{array}{l}\text { IPC-STG connection } \\
\text { (right) }\end{array}$ & Inhibitory \\
\hline & $\mathrm{B}(6,6)$ & .3703 & $>.9999$ & IPC self-inhibition & Inhibitory \\
\hline
\end{tabular}

634 Table 2. DCM parameter estimates. Posterior means are reported on a logarithmic scale, e.g.

635 a mean of -0.6631 corresponds to $\exp (-0.6631)=$ a decrease of the connection strength to $63651.53 \%$ of the baseline due to a given experimental factor.

637

638

639

640

641 
Akkal, D., Escola, L., Bioulac, B., Burbaud, P., 2004. Time predictability modulates pre-supplementary motor area neuronal activity. Neuroreport 15(8), 1283-1286.

Alink, A., Schwiedrzik, C.M., Kohler, A., Singer, W., Muckli, L., 2010. Stimulus Predictability Reduces Responses in Primary Visual Cortex. Journal of Neuroscience 30 (8), 2960-66.

Auksztulewicz, R., Friston, K., 2016. Repetition suppression and its contextual determinants in predictive coding. Cortex 80, 125-140.

Auksztulewicz, R., Friston, K.J., Nobre, A.C., 2017. Task relevance modulates the behavioural and neural effects of sensory predictions. PLoS Biol 15(12), e2003143.

Brown, H.R., Friston, K.J., 2012. Dynamic causal modelling of precision and synaptic gain in visual perception an EEG study. Neuroimage 63(1), 223-231.

Bueti, D., Bahrami, B., Walsh, V., Rees, G., 2010. Encoding of temporal probabilities in the human brain. $J$ Neurosci 30(12), 4343-52.

Chao, Z.C., Takaura, K., Wang, L., Fujii, N., Dehaene, S., 2018. Large-scale cortical networks for hierarchical prediction and prediction error in the primate brain. Neuron 100, 1-15.

Costa-Faidella, J., Baldeweg, T., Grimm, S., Escera, C., 2011. Interactions between 'What' and 'When' in the Auditory System: Temporal Predictability Enhances Repetition Suppression. Journal of Neuroscience 31 (50), 18590-97.

Coull, J.T., Cotti, J., Vidal, F., 2016. Differential roles for parietal and frontal cortices in fixed versus evolving temporal expectations: Dissociating prior from posterior temporal probabilities with fMRI. Neuroimage 141, 40-51.

Coull, J.T., Nazarian, B., Vidal, F., 2008. Timing, Storage, and Comparison of Stimulus Duration Engage Discrete Anatomical Components of a Perceptual Timing Network. Journal of Cognitive Neuroscience 20 (12), 2185-97.

Coull, J.T., Nobre, A.C., 1998. Where and When to Pay Attention: The Neural Systems for Directing Attention to Spatial Locations and to Time Intervals as Revealed by Both PET and FMRI. Journal of Neuroscience 18 (18), 7426-35.

Coull, J.T., Vidal, F., Nazarian, B., Macar, F., 2004. Functional Anatomy of the Attentional Modulation of Time Estimation. Science 303 (5663), 1506-8.

Cui, X., Stetson, C., Montague, P.R., Eagleman, D.M., 2009. Ready...Go: Amplitude of the FMRI Signal Encodes Expectation of Cue Arrival Time. PLOS Biology 7 (8), e1000167.

de Lange, F.P., Heilbron, M., Kok, P., 2018. How do expectations shape perception? Trends Cogn Sci 22(9), 764779.

Feldman, H., Friston, K.J., 2010. Attention, uncertainty, and free-energy. Front Hum Neurosci 4, 215.

Fischer, R., Plessow, F., Ruge, H., 2013. Priming of Visual Cortex by Temporal Attention? The Effects of Temporal Predictability on Stimulus(-Specific) Processing in Early Visual Cortical Areas. NeuroImage 66 (February), 261-69.

Friston, K., 2005. A Theory of Cortical Responses. Philosophical Transactions of the Royal Society of London B: Biological Sciences 360 (1456), 815-36.

Friston, K., Harrison, L., Daunizeau, J., Kiebel, S., Phillips, C., Trujillo-Barreto, N., Henson, R., Flandin, G., Mattout, J., 2008. Multiple sparse priors for the M/EEG inverse problem. Neuroimage. 39, $1104-1120$.

Friston, K.J., Litvak, V., Oswal, A., Razi, A., Stephan, K.E., van Wijk, B.C.M., Ziegler, G., Zeidman, P., 2016. Bayesian model reduction and empirical Bayes for group (DCM) studies. Neuroimage 128, 413-431.

Garrido, M.I., Kilner, J.M., Kiebel, S.J., Stephan, K.E., Baldeweg, T., Friston, K.J., 2009. Repetition Suppression and Plasticity in the Human Brain. NeuroImage 48 (1), 269-79.

Ghose, G.M., Maunsell, J.H., 2002. Attentional modulation in visual cortex depends on task timing. Nature 419(6907), 616-620.

Gilbert, J.R., Moran, R.J., 2016. Inputs to prefrontal cortex support visual recognition in the aging brain. Sci Rep 6, 31943.

Herbst, S.K., Fiedler, L., Obleser. J., 2018. Tracking Temporal Hazard in the Human Electroencephalogram Using a Forward Encoding Model. ENeuro 5 (2).

Hsu, Y.F., Hämäläinen, J.A., Waszak, F., 2013. Temporal Expectation and Spectral Expectation Operate in Distinct Fashion on Neuronal Populations. Neuropsychologia 51 (13), 2548-55.

Ille, N., Berg, P., Scherg, M., 2002. Artifact correction of the ongoing EEG using spatial filters based on artefact and brain signal topographies. J Clin Neurophysiol. 19, 113-124.

Janssen, P., Shadlen, M.N., 2005. A representation of the hazard rate of elapsed time in macaque area LIP. Nat Neurosci 8(2), 234-241.

Kanai, R., Lloyd, H., Bueti, D., Walsh, V., 2011. Modality-Independent Role of the Primary Auditory Cortex in Time Estimation. Experimental Brain Research 209 (3), 465-71. 
Kiebel, S.J., Daunizeau, J., Friston, K.J., 2008. A hierarchy of time-scales and the brain. Plos Comp Biol 4(11), e1000209.

Kilner, J.M., Kiebel, S.J., Friston, K.J., 2005. Applications of random field theory to electrophysiology. Neurosci Lett. 374, $174-178$.

Kok, P., Jehee, J.F.M., de Lange, F.P., 2012. Less Is More: Expectation Sharpens Representations in the Primary Visual Cortex. Neuron 75 (2), 265-70.

Lange, K., 2013. The Ups and Downs of Temporal Orienting: A Review of Auditory Temporal Orienting Studies and a Model Associating the Heterogeneous Findings on the Auditory N1 with Opposite Effects of Attention and Prediction. Frontiers in Human Neuroscience 7.

Lee, T.S., Mumford, D., 2003. Hierarchical Bayesian Inference in the Visual Cortex. JOSA A 20 (7), 1434-48.

Leon, M.I., Shadlen, M.N., 2003. Representation of time by neurons in the posterior parietal cortex of the macaque. Neuron 38(2), 317-327.

Litvak, V., Jha, A., Flandin, G., Friston, K. 2013. Convolution models for induced electromagnetic responses. Neuroimage 64(6), 388-398.

Miniussi, C., Wilding, E.L., Coull, J.T., Nobre, A.C., 1999. Orienting Attention in TimeModulation of Brain Potentials. Brain 122 (8), 1507-18.

Moran, R.J., Campo, P., Symmonds, M., Stephan, K.E., Dolan, R.J., Friston, K.J., 2013. Free energy, precision and learning: the role of cholinergic neuromodulation. J Neurosci 33(19), 8227-8236.

Moran, R.J., Jung, F., Kumagai, T., Endepols, H., Graf, R., Dolan, R.J., Friston, K.J., Stephan, K.E., Tittgemeyer, M., 2011. Dynamic causal models and physiological inference: a validation study using isoflurane anaesthesia in rodents. PLoS One 6(8), e22790.

Morillon, B., Baillet, S., 2017. Motor Origin of Temporal Predictions in Auditory Attention. Proceedings of the National Academy of Sciences 114 (42), E8913-21.

Muller, T., Nobre, A.C., 2014. Flow of Time: Perceiving the Passage of Time: Neural Possibilities. Annals of the New York Academy of Sciences 1326 (1), 60-71.

Nobre, A., Correa, A., Coull, J., 2007. The hazards of time. Curr Opin Neurobiol 17(4), 465-470.

Papadopoulou, M., Leite, M., van Mierlo, P., Vonck, K., Lemieux, L., Friston, K., Marinazzo, D., 2015. Tracking slow modulations in synaptic gain using dynamic causal modelling: validation in epilepsy. Neuroimage $107,117-126$.

Phillips, H. N., Blenkmann, A., Hughes, L. E., Kochen, S., Bekinschtein, T. A., Cam-Can, \& Rowe, J. B., 2016. Convergent evidence for hierarchical prediction networks from human electrocorticography and magnetoencephalography. Cortex 82, 192-205.

Pinotsis, D.A., Schwarzkopf, D.S., Litvak, V., Rees, G., Barnes, G., Friston, K.J., 2013. Dynamic causal modelling of lateral interactions in the visual cortex. Neuroimage 66, 563-576.

Rao, S.M., Mayer, A.R., Harrington, D.L., 2001. The Evolution of Brain Activation during Temporal Processing. Nature Neuroscience 4 (3), 317.

Recasens, M., Leung, S., Grimm, S., Nowak, R., Escera, S., 2014. Repetition suppression and repetition enhancement underlie auditory memory-trace formation in the human brain: an MEG study. Neuroimage $108,75-86$.

Segaert, K., Weber, K., de Lange, F.P., Petersson, K.M., Hagoort, P., 2013. The suppression of repetition enhancement: a review of fMRI studies. Neuropsychologia 51(1), 59-66.

Spitzer, B., Blankenburg, F., Summerfield, C., 2016. Rhythmic gain control during supramodal integration of approximate number. Neuroimage 129, 470-479.

Spratling, M.W., 2010. Predictive Coding as a Model of Response Properties in Cortical Area V1. Journal of Neuroscience 30 (9), 3531-43.

Stolk, A., Todorovic, A., Schoffelen, J.M., Oostenveld, R., 2013. Online and offline tools for head movement compensation in MEG. Neuroimage 68, 39-48.

Todorovic, A., Schoffelen, J.M., van Ede, F., Maris, E., de Lange, F.P., 2015. Temporal expectation and attention jointly modulate auditory oscillatory activity in the beta band. PLoS One 10(3), e0120288.

Todorovic, A., van Ede, F., Maris, E., de Lange, F.P., 2011. Prior Expectation Mediates Neural Adaptation to Repeated Sounds in the Auditory Cortex: An MEG Study. Journal of Neuroscience 31 (25), 9118-23.

Vallesi, A., McIntosh, A.R., Shallice, T., Stuss, D.T., 2009. When time shapes behaviour: fMRI evidence of brain correlates of temporal monitoring. J Cogn Neurosci 21(6), 1116-26.

Wacongne, C., Labyt, E., van Wassenhove, V., Bekinschtein, T., Naccache, L., Dehaene, S., 2011. Evidence for a Hierarchy of Predictions and Prediction Errors in Human Cortex. Proceedings of the National Academy of Sciences 108 (51), 20754-59.

Wiener, M., Turkeltaub, P.E., Coslett, H.B., 2010. Implicit Timing Activates the Left Inferior Parietal Cortex. Neuropsychologia 48 (13), 3967-71. 